\begin{tabular}{|c|l|}
\hline Title & Double network hydrogels from polyzwitterions : high mechanical strength and excellent anti-biofouling properties \\
\hline Author(s) & $\begin{array}{l}\text { Y in, Haiyan; A kasaki, Taigo; Sun, Tao Lin; Nakajima, Tasuku; Kurokawa, Takay uki; Nonoyama, Takay uki; Taira, } \\
\text { Toshio; Saruwatari, Y oshiyuki; Gong, Jian Ping }\end{array}$ \\
\hline Citation & $\begin{array}{l}\text { Journal of Materials Chemistry B, 1(30), 3685-3693 } \\
\text { https://doi.org/10.1039/c3tb20324g }\end{array}$ \\
\hline Issue Date & 2013 05-23 \\
\hline Doc URL & http://hdl.handle.net/2115/55723 \\
\hline Type & article (author version) \\
\hline File Information & manuscript.pdf \\
\hline
\end{tabular}

Instructions for use 


\section{Double network hydrogels from polyzwitterions: high mechanical strength and excellent anti-biofouling properties}

Haiyan YIN ${ }^{\mathrm{a}}$, Taigo AKASAKI ${ }^{\mathrm{b}}$, Tao Lin SUN ${ }^{\mathrm{a}}$, Tasuku NAKAJIMA ${ }^{\mathrm{c}}$, Takayuki KUROKAWA $^{\mathrm{c}}$, Takayuki NONOYAMA ${ }^{\mathrm{c}}$, Toshio TAIRA ${ }^{\mathrm{d}}$, Yoshiyuki SARUWATARI ${ }^{\mathrm{e}}$ and Jian Ping GONG ${ }^{\mathrm{c}^{*}}$

${ }^{\mathrm{a}}$ Graduate School of Science, Hokkaido University, Sapporo, 060-0810, Japan

${ }^{\mathrm{b}}$ Graduate School of Life Science, Hokkaido University, Sapporo, 060-0810, Japan

${ }^{\mathrm{c}}$ Faculty of Advanced Life Science, Hokkaido University, Sapporo, 060-0810, Japan

${ }^{\mathrm{d}}$ Primary Cell Co., Ltd., Kita 21 Nishi 12, Kita-ku, Sapporo, 001-0021, Japan

eOsaka Organic Chemical Industry Ltd., 1-7-20 Azuchi-machi, Chuo-ku, Osaka, 541-0052, Japan

*Corresponding author.

Tel/Fax: +81-11-706-2774, E-mail: gong@mail.sci.hokudai.ac.jp 


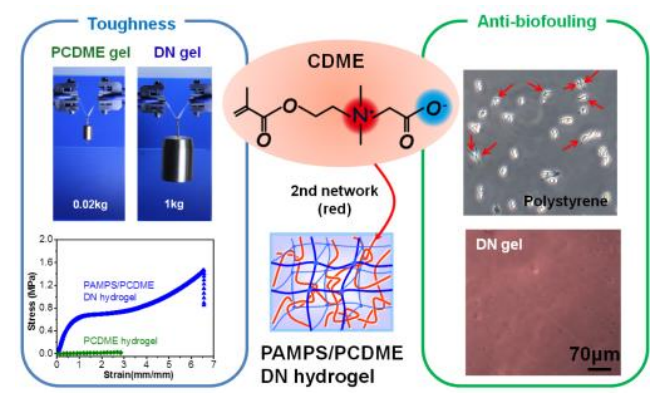

Novel hydrogels with high toughness and excellent anti-biofouling properties were synthesized from zwitterionic monomer based on double network (DN) concept. 


\section{Abstract}

Polyzwitterionic materials, which have both cationic and anionic groups in the polymeric repeat unit, show excellent anti-biofouling properties and are drawing more attention in the biomedical field. In this study, we have successfully synthesized novel single network hydrogels and double network (DN) hydrogels from the zwitterionic monomer, N-(carboxymethyl)-N, N-dimethyl-2-(methacryloyloxy) ethanaminium, inner salt (CDME). The polyCDME (PCDME) single network hydrogel behaves like a hydrophilic neutral hydrogel and its properties are not sensitive to temperature, $\mathrm{pH}$, or ionic strength over a wide range. DN hydrogels using the poly(2-acrylamido2-methylpropanesulfonic) (PAMPS) as the first network and PCDME as the second network, having a Young's modulus of $0.2 \sim 0.9 \mathrm{MPa}$, possess excellent mechanical strength (fracture stress: 1.2 1.4 MPa, fracture strain: 2.2 6.0 mm/mm) and toughness (work of extension at fracture: $0.9-2.4 \mathrm{MJ} / \mathrm{m}^{3}$ ) depending on the composition ratio of PCDME to PAMPS. The strength and toughness of the optimized PAMPS/PCDME DN is comparable to the normal PAMPS/PAAm DN hydrogels that use poly(acrylamide)(PAAm) as the second network. By macrophage adhesion test, both the PCDME hydrogels and the PAMPS/PCDME DN hydrogels have shown excellent anti-biofouling properties. These results demonstrate that the PCDME-based DN hydrogels have a high potential as a novel soft and wet biomaterial. 
Keywords: Zwitterion / double network hydrogel / high mechanical strength / anti-biofouling / macrophage 


\section{Introduction}

Anti-biofouling materials, which resist protein adsorption or cell adhesion, have widespread demand for various biomedical applications such as contact lens ${ }^{1}$, endoscope $^{2}$, artificial organs implants ${ }^{3}$, plasters for wound healing ${ }^{4}$, and biosensors. ${ }^{5}$ Various hydrophilic anti-biofouling materials, such as polyethylene glycol (PEG) ${ }^{6,7}$, zwitterionic polymers of phosphobetaine $(\mathrm{PB})^{8}$, sulfobetaine $(\mathrm{SB})^{9}$, and carboxybetaine $(\mathrm{CB})^{10}$ have been presented. As PEG is subject to oxidation ${ }^{11}$, which reduces its utility for wide application, zwitterionic polymers attract rising attention for their stability and excellent anti-biofouling properties. Ishihara et al. reported that the amount of protein adsorbed on the polysulfone/2-methacryloyloxyethyl phosphorylcholine (PSf/MPC) polymer blend membrane was significantly decreased with an increase in the composition of the blended $\operatorname{MPC}($ a type of $\mathrm{PB})$ polymer $^{12}$, and the inflammatory reaction of adherent cells was slightly reduced in the case of MPC-blended DL-lactide/glycolide copolymer (PLGA), comparing with the non-treated PLGA. ${ }^{13}$ Jiang et al. demonstrated that both poly(sulfobetaine methacrylate) (pSBMA) and poly(carboxybetaine methacrylate) (pCBMA) grafted on glass surfaces via surface-initiated atom transfer radical polymerization (ATRP) have excellent resistance to protein adsorption and mammalian cell adhesion. ${ }^{14}$ It was also reported that a 
zwitterionic poly(2-carboxy-N,N-dimethyl-N-[2'-(methacryloyloxy) ethyl] ethanaminium) (pCBMA-2) surface can prevent the attachment of proteins and microorganisms, and reduce the formation of biofilm on the surface. ${ }^{15}$ Kitano et al. have studied anti-biofouling properties of poly(N-(carboxymethyl)-N,N-dimethyl2-(methacryloyloxy) ethanaminium, inner salt) (PCDME, also named as PCMB in ref. 16, Scheme 1a), which is a novel CB type zwitterionic polymer that has a negatively charged $\mathrm{COO}^{-}$group and a positively charged $\mathrm{R}_{4} \mathrm{~N}^{+}$group. They have shown that the poly(CMB-co-BMA) film from random copolymerization of $\mathrm{CMB}$ and $n$-butyl methacrylate(BMA) significantly enhances wound closure and complete healing of a full-thickness excisional wound compared with the effect of PBMA and poly(ethylene terephthalate)(PET) films ${ }^{4}$. Moreover, the amount of protein adsorption and number of cell adhered onto the poly(CMB-co-BMA) copolymers was smaller than those of other polymers. ${ }^{16}$ In addition, they also reported that PCMB-protected gold nanoparticles resist against non-specific adsorption of proteins. ${ }^{17} \mathrm{CB}$ type zwitterionic materials have received wide attention due to their simple synthesis process and possibility of modification with biomacromolecules such as fibronectin to control the aortic endothelial cell adhesion. ${ }^{18}$

Hydrogels are also attractive scaffold materials because their mechanical properties 
can be tailored to mimic those of natural tissues, and are useful for regenerative medicine such as barriers, drug delivery carriers, and cell encapsulation. ${ }^{19}$ Due to the excellent anti-biofouling properties of zwitterionic materials, several groups attempted to synthesize zwitterionic hydrogels. ${ }^{20-23}$ Jiang et al. prepared zwitterionic hydrogels such as poly(sulfobetaine methacrylate) (pSMBA) (a type of SB) and poly(carboxybetaine methacrylate) (pCBMA) (a type of $\mathrm{CB}$ ) which shows excellent resistance to BAEC adhesion in vitro. ${ }^{22}$ However, these conventional hydrogels with single networks are too weak and brittle, limiting their application. ${ }^{24,25}$ For the biomaterials-based tissue engineering strategies, mechanical compatibility (modulus matching with other soft tissues) and durability (high strength and toughness) are important criteria for the materials. For example, the Young's modulus and the tensile strength of human arteries are $0.88 \mathrm{MPa}$ and $0.35 \mathrm{MPa}$ respectively. ${ }^{26}$ Several strategies to toughen hydrogels have been introduced such as interpenetrating network structure ${ }^{27}$, homogeneous network structure ${ }^{28}$, and formulating composites with clay. ${ }^{29}$ Among the several approaches adopted for strengthening hydrogels, development of double-network gels (DN gels), the toughest and strongest hydrogels having both cartilage-like robustness and high water content (ca. $90 \mathrm{wt} \%$ ), is the most notable. ${ }^{30}$ Tough DN hydrogels consist of two interpenetrating networks with a contrasting 
topological structure: one network is rigid and brittle, composed of short and extended chains (high cross-linker density) in dilute concentration while the other network is soft and ductile, composed of very long chains in high concentration. ${ }^{31}$ This topological structure is initially created by a two-step sequential polymerization in which the rigid network was synthesized first using polyelectrolyte poly(2-acrylamido-2-methylpropanesulfonic acid) (PAMPS, Scheme 1b), followed by the soft network being synthesized using neutral polyacrylamide (PAAm). The PAMPS/PAAm DN gel is denoted as a normal DN gel in this work. The DN principle for toughening of hydrogels is universal, and can be applied to any kind of polymer combination. ${ }^{32}$ Thus, double network principle provides a general pathway to develop hydrogels with cartilage-like robustness and high water content (ca. $90 \mathrm{wt} \%)$.

The purpose of this study is to prepare anti-biofouling hydrogels with high strength and toughness using zwitterionic polymers of PCDME. We first synthesized the PCDME single network hydrogels to confirm its physical properties. As PCDME hydrogels have both negatively charged $-\mathrm{COO}^{-}$groups and positively charged $-\mathrm{R}_{3} \mathrm{~N}^{+}$ groups, they behave like neutral hydrogels, as confirmed by the relationship between Young's modulus and swelling degree, as well as its behavior in $\mathrm{NaCl}$ aqueous solution. This result promises that the PCDME can be used as the second network of tough DN 
gels. Thus, we prepared tough PAMPS/PCDME DN hydrogels using DN concept. We confirmed that the toughness of the PAMPS/PCDME DN hydrogels are comparable to the conventional tough DN hydrogels consisting of poly(2-acrylamido-2methylpropanesulfonic acid) (PAMPS) as the first network and polyacrylamide (PAAm) as the second network. The anti-biofouling properties of the PCDME single network hydrogel and PAMPS/PCDME DN hydrogel have been confirmed by the macrophage adhesion test. We have chosen the macrophage adhesion test to investigate the antifouling properties since the inflammatory reaction occurs through the macrophage adhesion on the implant surfaces.

\section{Experimental}

\section{Materials}

$N$-(carboxymethyl)- $N, N$-dimethyl-2-(methacryloyloxy)ethanaminium, inner salt (CDME, Osaka Organic Chemical Co., Ltd., Japan) and 2-acrylamido-2-methylpropane -sulfonic acid (AMPS, Toa Gosei Co., Ltd., Japan) were used as received. $N, N$ '-Methylenebis(acrylamide) (MBAA, Wako Pure Chemical Industries, Ltd., Japan) was recrystallized from ethanol. 2-Oxoglutaric acid (Wako Pure Chemical Industries, Ltd.) was used as received. Acrylamide (AAm, Junsei Chemical Co., Ltd., Japan) was 
recrystallized from chloroform.

\section{Methods}

\subsection{Synthesis of hydrogel}

The single network PCDME hydrogels were synthesized by photo-polymerization of precursor aqueous solutions composed of 1-3 $\mathrm{M}$ of monomer CDME, 0.3-6 mol\% of cross-linker MBAA, and $0.1 \mathrm{~mol} \%$ of initiator 2-oxoglutaric acid (both relative to the monomer). When the CDME monomer concentration was changed from $1 \mathrm{M}$ to $3 \mathrm{M}$, the $\mathrm{pH}$ value of the precursor solutions changed from 4.53 to 5.58. These aqueous solutions were photo-polymerized by UV irradiation for $8 \mathrm{~h}$ in an argon blanket. After synthesis of the hydrogels, they were put in pure water for 1 week until swelling equilibrium was reached, and the samples were cut to cylindrical shape of $15 \mathrm{~mm}$ diameter by a gel cutter for the measurement of swelling ratio and compressive test. From the results we selected optimized conditions that are suitable for hydrogel formation. The single network PCDME hydrogels were coded as SNx-y, here the x and y stand for the CDME concentration $\mathrm{C}_{\mathrm{CDME}}(\mathrm{M})$ and the MBAA cross-linker density $\mathrm{C}_{\mathrm{MBAA}}(\mathrm{mol} \%)$ respectively. The compositions of samples synthesized are shown in

\section{Table 1.}


Using the DN concept we prepared tough PAMPS/PCDME DN gels by two-step sequential free-radical polymerization. ${ }^{30}$ In the first step, poly(2-acrylamido-2methylpropanesulfonic acid)(PAMPS) hydrogels were prepared by photo polymerization from $1 \mathrm{M}$ of AMPS, $4 \mathrm{~mol} \%$ of cross-linker MBAA, and $0.1 \mathrm{~mol} \%$ initiator using UV irradiation for $8 \mathrm{~h}$ in an argon blanket. In the second step, the prepared PAMPS hydrogels were immersed into the aqueous solutions containing 1-3 $\mathrm{M}$ of CDME, $0.02 \mathrm{~mol} \%$ of MBAA and $0.01 \mathrm{~mol} \%$ initiator (both were in relative to CDME monomer) for at least 3 days. The PCDME second network was synthesized in the presence of PAMPS gels by UV irradiation. After this 2-step synthesis, the PAMPS/PCDME DN hydrogels were immersed in pure water for 1 week until reaching swelling equilibrium.

The PAMPS/PCDME DN hydrogels were coded as DNx, where, $\mathrm{x}$ stands for the CDME concentration $(\mathrm{M})$ of the second network. For comparison, the normal PAMPS/PAAm DN hydrogel was also prepared at 2M AAm, and it was denoted as n-DN2. The compositions of samples synthesized are shown in Table 2.

\subsection{Swelling degree measurement}

After reaching swelling equilibrium, the weights of hydrogels in swollen and dried 
states were measured with a moisture balance MOC-120H (Shimadzu co.). Swelling degree $q$ was determined as $q=w / w_{0}$, where $w$ and $w_{0}$ are the weight of swollen gel and the dried sample, respectively. The water content $\mathrm{c}$ was estimated as $c=(1-1 / q) \mathrm{x} 100 \%$. The swelling behaviors of PCDME hydrogels and PAMPS/PCDME DN hydrogels in various concentrations of $\mathrm{NaCl}$ aqueous solutions or $\mathrm{pH}$ solutions were characterized by the volume ratio $\mathrm{V} / \mathrm{V}_{0}$, where $\mathrm{V}$ and $\mathrm{V}_{0}$ were volumes of the samples in $\mathrm{NaCl}$ or $\mathrm{pH}$ solutions and in pure water, respectively.

\subsection{Mechanical properties}

1) Compressive test

Mechanical properties of PCDME single network hydrogels were measured by compressive testing using a tensile-compressive tester (Tensilon RTC-1310A, Orientec Co.). Hydrogel samples with a $15 \mathrm{~mm}$ diameter cylindrical shape were placed at the center of the lower compression plate. The sample was then compressed by the upper plate, which was connected to a $100 \mathrm{~N}$ load cell, at a velocity of $0.1 \mathrm{~mm} / \mathrm{min}$.

2) Tensile test

Mechanical properties of PCDME hydrogels and PAMPS/PCDME DN hydrogels 
were measured by tensile testing using a tensile tester (Instron 5965, Instron Co.). For the tensile test, samples of $1.34 \sim 2.5 \mathrm{~mm}$ in thickness were cut into a dumbbell shape (width of $2 \mathrm{~mm}$, length of $12 \mathrm{~mm}$ ) using a gel cutter (JIS-K6251-7). Tensile velocity was $50 \mathrm{~mm} / \mathrm{min}$.

For both compressive test and tensile test, the fracture stress and the fracture strain were determined as the nominal stress and the nominal strain at the failure point, respectively. Young's modulus $E$ was also determined as the slope at the strain range of 0-0.1 from the stress-strain curve. Work of extension was determined from the area under the stress-strain curve of tensile test. Measurements were performed at least three times for each sample.

\subsection{Surface properties}

The static contact angles to water, $\theta$, of the hydrogels and TCPS substrate were measured with a Drop Master 300 (Kyowa Interface Science Co., Ltd) in air at room temperature. The volume of the water droplet $(2 \mu \mathrm{l})$ was maintained constant for all the substrates, and the gravity effect can be neglected. The water on the surface of hydrogels was removed slightly with kimwipe before measurements. The $\theta$ data in Table 3 were the averages of 10 measurements for each sample. 


\subsection{Macrophage culture}

In order to investigate cell adhesion, RAW 264.7 macrophages (Primary Cell Co. Ltd. Japan) were used. The hydrogel samples for the adhesion test were equilibrated in HEPES buffer solution (HEPES: $5 \times 10^{-3} \mathrm{M}, \mathrm{NaHCO}_{3}: 1.55 \times 10^{-2} \mathrm{M}, \mathrm{NaCl}: 0.14 \mathrm{M}, \mathrm{pH}$ 7.4 and Phenol red: $2.5 \times 10^{-3} \mathrm{~g} / \mathrm{L}$ ) for one week by exchanging the buffer solution every day. The hydrogel samples, cut into a disc-shape of $15.4 \mathrm{~mm}$, were sterilized at $120^{\circ} \mathrm{C}$ for 20 min. The macrophages were cultured in Dulbecco's Modified Eagle Medium (DMEM) supplemented with $10 \%$ fetal bovine serum and seeded on different kinds of hydrogel surfaces such as PCDME, PAMPS/PCDME DN, and tissue culture polystyrene (TCPS) as a control at a density of $5.0 \times 10^{4}$ cells $/ \mathrm{cm}^{3}$ in a $5 \% \mathrm{CO}_{2}$ humidified atmosphere at $37^{\circ} \mathrm{C}$. Macrophages were allowed to attach for $24 \mathrm{~h}$. After that, the non-adherent cells were removed by rinsing with PBS solution. Images of the cells adhered on the surfaces were obtained with a phase contrast microscope (OLYMPUS CKX31, Japan) equipped with a digital camera using a $4 \mathrm{x}$ objective lens. The numbers of cells adhered on the surfaces were determined from three images taken from 3 samples and were expressed as cells per $\mathrm{mm}^{2}$. For each hydrogels, at least two runs of experiments were performed. 


\section{Results and Discussion}

1. PCDME single network hydrogels

As shown in Table 1, the Young's modulus $E$ increases, while the swelling degree $q$ decreases with an increase in MBAA crosslinker density for the gels at the equilibrium swelling state in water. At the same MBAA density, $E$ shows an increase and $q$ shows a decrease tendency when the CDME monomer concentration increases from $1 \mathrm{M}$ to $3 \mathrm{M}$.

The Young's modulus of a hydrogel has the same origin of rubber elasticity, and it is the product of the number of elastically effective chains in the unit volume $v_{e}$ and the energy for stretching a chain $E_{\text {energy, }}$, i.e., $E=3 v_{e} E_{\text {energy }}{ }^{33}$ For chains in random coil conformation, $E_{\text {energy }}=k_{B} T$, where $k_{B}$ is the Boltzmann constant and $T$ is the temperature. However, when the chain is initially in a highly stretched state, such as a chain of a highly swollen polyelectrolyte hydrogel, further stretching of the chain needs more energy. Therefore, $E_{\text {energy }}$ is larger than $k_{B} T$. This means that with an increase in the degree of swelling, $v_{e}$ decreases while $E_{\text {energy }}$ has a tendency to increase. For a neutral gel that modestly swells in water, the former effect is dominant. On the other hand, for a strong polyelectrolyte gel that substantially swells in water, the latter effect becomes dominant. As $q$ is roughly the inverse of polymer volume fraction, $E \sim q^{-9 / 4}$ and $E \sim q^{-1}$ for neutral hydrogels and polyelectrolyte hydrogels in water, respectively, at the 
equilibrium swelling, regardless their preparation condition. ${ }^{33,34}$ Therefore the modulus $E$ of a hydrogel is directly related to the swelling degree $q$.

To elucidate whether the PCDME hydrogels behave like neutral gels or polyelectrolyte gels, we plot Young's modulus $E$ against the swelling degree $q$ of the hydrogel synthesized at different cross-linker density and monomer concentration of the precursor solution (Figure 1). We found that the $E$ and $q$ follows the same scaling relationship of $E \sim q^{-2.15}$, regardless their preparation conditions. The exponent -2.15 is very close to the theoretical value of $-9 / 4$ for a neutral hydrogel. This result indicates that the PCDME hydrogels, having both the negatively charged $-\mathrm{COO}^{-}$group and positively charged $\mathrm{R}_{3} \mathrm{~N}^{+}$group, behave as a hydrophilic neutral hydrogel in water. The partial chains of PCDME hydrogels are in a random coil conformation. This result permitted us to estimate the molecular weight $M_{c}(\mathrm{~g} / \mathrm{mol})$ between cross-linking points using the rubber elasticity theory. ${ }^{35}$

$$
E=3 v_{e} k_{B} T=\frac{3 \phi \rho R T}{M_{c}}
$$

Where $\phi$ is the polymer volume fraction, $\rho$ is the polymer density in a dry state $\left(\mathrm{g} / \mathrm{m}^{3}\right)$ (assumed as $1.7 \mathrm{~g} / \mathrm{m}^{3}$ ), and $\mathrm{R}$ is the gas constant. The number of repeat units between the crosslinking points $N$ estimated from $M_{c}$ and the CDME monomer molecular weight $(233.33 \mathrm{~g} / \mathrm{mol})$ are shown in Table 1. 
Some zwitterion polymers do not dissolve in water at low temperature and show upper critical solution temperature (UCST). ${ }^{36}$ For example, the zwitterionic polymer, poly-3-dimethyl(methacryloyloxyethyl)ammonium propane sulfonate (PDMAPS), having a very similar structure to PCDME, showed UCST that is strongly dependant on its molecular weight and the salt concentration of the aqueous media. ${ }^{37}$ The PDMAPS gel reversibly responds to changing the temperature and the $\mathrm{NaCl}$ solution. ${ }^{38}$ However, we found that the PCDME hydrogels are transparent over the temperature range of $0-80^{\circ} \mathrm{C}$, indicating that PCDME hydrogels are very stable and have no phase separation in this wide range of temperature. We also found that both the modulus $E$ and the swelling ratio $V / V_{0}$ of PCDME hydrogels are insensitive to the ionic strength over a wide range of $\mathrm{NaCl}$ concentration $\left(10^{-4} \mathrm{M}-3.16 \mathrm{M}\right)$, as shown in Figure 2a. Here $V$ and $V_{0}$ are the volume of the sample in $\mathrm{NaCl}$ aqueous solution and in water, respectively. This behavior is also quite different from the PDMAPS that shows a strong salt dependence of the solution behavior. ${ }^{38}$

As the $-\mathrm{COO}^{-}$group is a weak acid, it can associate with $\mathrm{H}^{+}$ions at low $\mathrm{pH}$ to form $-\mathrm{COOH}$. On the other hand, the $-\mathrm{R}_{3} \mathrm{~N}^{+}$group has the quaternized amine that is not sensitive to $\mathrm{pH}$ change. Therefore the PCDME hydrogels may behave like a positively charged polyelectrolyte gel at low $\mathrm{pH}$, and swell substantially. The $\mathrm{pH}$-dependence of 
the PCDME hydrogels is shown in Figure 3. For comparison, the result for a poly(acrylic acid) (PAAc) hydrogel, which deswells at low $\mathrm{pH}$ due to protonation of the $-\mathrm{COO}^{-}$group, is also shown in the Figure 3. We observed that the on-set of swelling of the PCDME hydrogels starts around $\mathrm{pH} 3$, much lower than the on-set of deswelling of PAAc hydrogel that is around $\mathrm{pH} 6$. This indicates that the association of $-\mathrm{COO}^{-}$with $\mathrm{H}^{+}$is substantially suppressed in PCDME hydrogels in comparison with that of PAAc hydrogels. The stability of PCDME hydrogels in a wide range of temperature, ionic strength, and $\mathrm{pH}$ is favorable for the application of this material in physiological environments.

\section{Tough PAMPS/PCDME DN hydrogels}

The single network PCDME hydrogels were mechanically weak and showed very brittle character (Table 1). For example, the PCDME hydrogel (SN3-0.3) broke at very low compressive stress $(0.6 \mathrm{MPa})$ and at very small compressive strain $(1 \mathrm{~mm} / \mathrm{mm})$. In order to improve the mechanical strength of the zwitterionic PCDME hydrogel, we have used the DN concept to prepare PAMPS/PCDME DN hydrogels, where the first network PAMPS is a negatively charged polyelectrolyte.

As revealed in our previous studies, the tough PAMPS/PAAm DN hydrogels (normal 
DN gels) have an interpenetrated network structure with contrasting features, i.e., the first network is brittle with short chains in low concentration and the second network is ductile with long chains in high concentration. In this setting the optimum molar ratio of the $2^{\text {nd }}$ network to the $1^{\text {st }}$ network is around 20 for materials with the extraordinary toughness. This contrasting topological structure is formed owing to the substantial swelling of the first polyelectrolyte PAMPS hydrogel in aqueous solution of the precursor solution of the second neutral PAAm network. ${ }^{30,32}$ We first study the swelling behavior of the PAMPS hydrogel in precursor solutions of the PCDME second network which containing 1, 2 and $3 \mathrm{M}$ of CDME monomer, $0.02 \mathrm{~mol} \%$ of MBAA, and 0.01 mol\% initiator with respect to the monomer concentration. As shown in Figure 4, the as-prepared PAMPS hydrogel swelled substantially in the precursor solutions, and the volume swelling ratio $\mathrm{V} / \mathrm{V}_{0}$ is similar to that in water, hardly dependent on the CDME concentration. Here, $\mathrm{V}_{0}$ is the volume of the PAMPS hydrogel in the as-prepared state, and V is volume of the PAMPS hydrogel in pure water, various CDME solutions, or in 2 M AAm solution. This confirms again that CDME behaves like a neutral monomer, and PCDME network can be induced in the PAMPS hydrogel with a high concentration.

Table 2 shows the composition and properties of PAMPS/PCDME DN hydrogels prepared at various CDME concentrations of the second network precursor solution, 
while keeping the cross-linker density of the second network a constant $(0.02 \mathrm{~mol} \%)$. The water content $c$ of PAMPS/PCDME DN hydrogels is about $80 \mathrm{wt} \%$, slightly decreased with the increase in the CDME concentration of the precursor solution. This water content is lower than that of the normal PAMPS/PAAm DN hydrogel (n-DN2: c $90 \mathrm{wt} \%$ ), which can be understood by the higher molecular weight of the CDME monomer $\left(233.33 \mathrm{~g} \mathrm{~mol}^{-1}\right)$ than that of AAm $\left(71.08 \mathrm{~g} \mathrm{~mol}^{-1}\right)$. From the elemental analysis of the PAMPS/PCDME DN hydrogels, the molar ratio of PCDME to PAMPS was 10,25 , and 38 for concentration of 1,2 , and $3 \mathrm{M}$ CDME solutions, respectively (Table 2).

Figure 5 demonstrates that the weak single network PCDME hydrogel (SN1) can only sustain a load of $0.02 \mathrm{kgf}$ while a tough PAMPS/PCDME DN hydrogel (DN3) can sustain a large load 1kgf. Both of them have the same size, $20 \times 4 \times 2.5 \mathrm{~mm}^{3}$. Figure 6a presents the tensile stress-strain curves of PAMPS/PCDME DN hydrogels. With the increase of $\mathrm{CDME}$ concentration from $1 \mathrm{M}$ to $3 \mathrm{M}$, the Young's modulus increased by more than a factor of four, from $0.2 \mathrm{MPa}$ to $0.9 \mathrm{MPa}$. With the increase in CDME concentration, the fracture stress only increased slightly. However, the fracture strain and the work of extension at fracture increased remarkably (Table 2). Sample DN2 and DN3 of PAMPS/PCDME DN hydrogels, having molar ratios of $2^{\text {nd }} / 1^{\text {st }}$ close to the 
optimized condition, show similarly high toughness as the normal PAMPS/PAAm DN hydrogel (Figure 6a). Specifically, the fracture stress of the PAMPS/PCDME DN hydrogel (DN3) was 60 times higher than that of the PCDME single network hydrogel(SN3-0.3).

As shown in Figure 6b, the tensile behaviors of the DN hydrogels do not change with the tensile velocity. This indicates the negligible viscous effect during the deformation, in agreement with PAMPS/PAAm DN hydrogels reported. ${ }^{39}$ Previously, we have confirmed that the mechanical performance of DN hydrogels does not change with the sample thickness, and ultrathin double network (UTDN) hydrogels of about $\sim 100 \mu \mathrm{m}$ thickness also shows similar high mechanical strength and toughness to the bulk DN hydrogels. ${ }^{40}$ As the elasticity of the DN hydrogels comes from the densely crosslinked PAMPS first network, the increase in the elastic modulus of the DN hydrogels with CDME concentration (Table 2) suggests the increase in the stiffness of the PAMPS chains. To confirm this, we further measured the swelling of the DN hydrogels in water. As shown in Figure 4, after the second polymerization, in comparison with the volume of PAMPS hydrogel in the corresponding monomer solution, the volume $V$ of PAMPS/PCDME DN hydrogels in water further increases for $\mathrm{C}_{\mathrm{CDME}}=2 \mathrm{M}$ and $3 \mathrm{M}$. This indicates that the loosely cross-linked PCDME network exerts additional osmotic 
pressure to the PAMPS network, and the PAMPS network that was already in relatively extended state swells even further. As a result, the stiffness of each PAMPS chain further increases, which leads to the increase in the modulus of the DN hydrogels. This explanation also agrees with the fact that the PAMPS/PCDME DN hydrogel prepared at $1 \mathrm{M} \mathrm{CDME}$ and the PAMPS/PAAm gel prepared at $2 \mathrm{M}$ AAm both do not exhibiting large further swelling after the second polymerization (Figure 4), showed the relatively low modulus of 0.2 MPa (Table 2).

Thus, a great improvement in the mechanical properties of PAMPS/PCDME DN hydrogels has been obtained through the combination of rigid polyelectrolyte PAMPS and flexible zwitterion polymer PCDME. To validate the biological applicability of PAMPS/PCDME DN hydrogels, tensile tests have been performed after HEPES buffer washing and thermal sterilization. The results in Figure 6c show that the high elastic modulus and the yielding behavior of the PAMPS/PCDME DN hydrogel (DN3) do not change with these treatments. However, the fracture strain and stress decrease after treatment with sterilization. Even with some thermal degradation, the DN3 is still a very high strength hydrogel, and its mechanical properties (Young`s modulus $\sim 0.9 \mathrm{MPa}$, tensile strength $\sim 0.4 \mathrm{MPa}$, and tensile strain $\sim 2.5 \mathrm{~mm} / \mathrm{mm}$ ) match with the mechanical requirements of many applications for biomaterials. For example, the Young's modulus 
and the tensile strength of human arteries are $0.88 \mathrm{MPa}$ and $0.35 \mathrm{MPa}$, respectively, and these DN hydrogels match these properties well. ${ }^{26}$

We further confirmed that the tough PAMPS/PCDME DN hydrogels also showed insensitivity in their degree of swelling and modulus to changes in ionic strength (Figure 2b) and pH (Figure 3), similar to the PCDME single network hydrogel. The insensitivity of DN hydrogels to ionic strength indicates that the PAMPS first network is fully filled with the PCDME that exerts a high osmotic pressure to prevent the deswelling of the polyelectrolyte PAMPS in salt solution.

3. Macrophage adhesion test

Macrophage adhesion tests show that both the PCDME hydrogels and the tough PAMPS/PCDME DN hydrogels have the same excellent anti-biofouling properties. Figure 7 shows the typical behaviors of macrophages on the PCDME single network hydrogel(SN1-1), PAMPS/PCDME DN hydrogel(DN1), and TCPS as a control. After 24h macrophage seeding, no cell adhesion was observed on both the PCDME gel and the PAMPS/PCDME DN gel, while some macrophages adhered on the surface of TCPS (Figure 7a). After 24h, the number of adhered macrophage increased on the TCPS surface with a density of $145 \pm 13$ cells $/ \mathrm{mm}^{2}$, but there are no adhered cells on the two 
kinds of hydrogels with various compositions (Figure 7b). We also confirmed that no adhesion occurred on these hydrogels even after 7 days but the cell numbers increased on the surface of hydrogels. The cells proliferated on the hydrogels were collected and sub-cultured on the TCPS substrate. These sub-cultured cells showed similar adhesion behaves on the TCPS substrates. These results confirm that the PCDME-based hydrogels are non-toxic.

The non-toxicity of hydrogels using MBAA as crosslinker has also been confirmed by several in vivo studies, although MBAA monomer is toxic. For example, tough PAMPS/PDMAAm(poly(N,N'-dimethylacrylamide)) DN hydrogels using the MBAA as crosslinker do not cause inflammation when they were implanted in subcutaneous tissues. ${ }^{41}$ Furthermore, PAMPS/PDMAAm DN hydrogels implanted in the defected knee of joints induce spontaneous articular cartilage regeneration in vivo. ${ }^{42}$

Some studies on the relationship between the cell adhesion and wettability of the substrate have shown that the maximum cell adhesion occurs on substrates with a contact angle to water around $70^{\circ}$. Substrates with a contact angle deviating from this value, either more hydrophilic or more hydrophobic, show much less cell adhesion. ${ }^{43}$ Following this line, we measured contact angles of TCPS and hydrogels used for the macrophage adhesion test, and the result is shown in Table 3. The contact angle of 
TCPS substrate is around $70^{\circ}$ and the contact angles of the PCDME hydrogels and PAMPS/PCDME DN hydrogels vary from 11.4 to $37.5^{\circ}$, which increases with the decrease in the swelling degree $q$ of the hydrogels. The latter behavior indicates that the contact angle of the hydrogels increases with the polymer concentration that is proportional to 1/q. Accordingly, the behaviors of macrophages on the TCPS surface that shows high adhesion and on the PCDME and PAMPS/PCDME DN hydrogels that show no adhesion is basically in agreement with the $70^{\circ}$ phenomenological rule. It should be noted that, although the contact angle of the hydrogels increases with the polymer concentration, all the hydrogels show no adhesion. This indicates that cell adhesion not only depends on the contact angle but also depends on the chemical structure of the surface. Previously, researchers have revealed, by using Raman techniques, that carboxybetaine polymers do not disturb the structure of water absorbed on the polymer surface, and the excellent blood-compatibility of the carboxybetaine polymers has been related to this specific hydration state. ${ }^{44,45}$

As previously elucidated, the negatively charged hydrogels have adhesive interaction with cells. ${ }^{46,47}$ Why can the tough PAMPS/PCDME DN hydrogels can maintain the anti-biofouling property while containing the negatively charged PAMPS? According to Table 2, PCDME is the dominant component of the PAMPS/PCDME DN hydrogels. 
This may account for the negligible effect of PAMPS on the surface properties of the PAMPS/PCDME DN hydrogel.

\section{Conclusions}

We have successfully synthesized both single network hydrogels and double network hydrogels from the zwitterion monomer CDME. The PCDME hydrogels behaved as a hydrophilic neutral hydrogel. The swelling degree and modulus of the PCDME hydrogels were insensitive to the change in temperature, $\mathrm{pH}$, and ionic strength over a wide range. The PAMPS/PCDME DN hydrogels, containing $~ 80 \mathrm{wt} \%$ of water, showed similar toughness to the normal PAMPS/PAAm DN gel. The macrophage adhesion test confirms an excellent anti-biofouling property of PCDME hydrogels and the tough PAMPS/PCDME DN hydrogels. These results demonstrate that the PCDME-based DN hydrogels have a high potential as a novel soft and wet biomaterial.

\section{Acknowledgements}

This research was financially supported by a Grant-in-Aid for Scientific Research (S) (No. 124225006) from Japan Society for the Promotion of Science (JSPS). 


\section{Notes and References}

1 S. L. Willis, J. L. Court, R. P. Redman, J. H. Wang, S. W. Leppard, V. J. O'Byrne, S.

A. Small, A. L. Lewis, S. A. Jones and P. W. Stratford, Biomaterils, 2001, 22, $3261-3272$.

2 K. Marion, J. Freney, G. James, E. Bergeron, F. N. R. Renaud and J. W. Costerton, J.

Hospital. Infection., 2006, 64, 136-142

3 B. D. Ratner and S. J. Bryant, Annu. Rev. Biomed. Eng., 2004, 6, 41-75.

4 S. Fujishita, C. Inaba, S. Tada, M. Gemmei-Ide, H. Kitano and Y. Saruwatari, Biol. Pharm. Bull., 2008, 31, 2309-2315.

5 W. Yang, H. Xue, L. R. Carr, J. Wang and S. Jiang, Biosens. Bioelectron., 2011, 26, 2454-2459.

6 F. Zhang, E. T. Kang, K. G. Neoh, P. Wang and K. L. Tan, J. Biomed. Mater. Res., 2001, 56, 324-332.

7 S. H. Y. Cheo, P. Wang, K. L. Tan, C. C. Ho and E. T. Kang, J Mater Scien: Mater In Medic., 2001, 12, 377-384.

8 W. Feng, J. L. Brash and S. P. Zhu, Biomaterials, 2006, 27, 847-855.

9 L. Carr, G. Cheng, H. Xue and S. Jiang, Langmuir, 2010, 26, 14793-14798. 
10 Z. Zhang, H. Vaisocherova, G. Cheng, W. Yang, H. Xue and S. Jiang, Biomacromolecules, 2008, 9, 2686-2692.

11 D. A. Herold, K. Keil and D. E. Bruns, Bioche. Pharma., 1989, 38, 73-76.

12 K. Ishihara, K. Fukumoto, Y. Iwasaki and N. Nakabayashi, Biomaterials, 1999, 20, 1553-1559.

13 Y. Iwasaki, S. Sawada, K. Ishihara, G. Khang and H. B. Lee, Biomaterials, 2002, 23, 3897-3903.

14 Z. Zhang, T. Chao, S. Chen and S. Jiang, Langmuir, 2006, 22, 10072-10077.

15 G. Cheng, H. Xue, Z. Zhang, S. Chen and S. Jiang, Angew. Chem. Int. Ed., 2008, 47, $8831-8834$

16 S. Tada, C. Inaba, K. Mizukami, S. Fujishita, M. Gemmei-Ide, H. Kitano, A. Mochizuki, M. Tanaka and T. Matsunage, Macromol. Biosci., 2009, 9, 63-70.

17 K. Matsuura, K. Ohno, S. Kagaya and H. Kitano, Macromol. Chem. Phys., 2007, 208, $862-873$.

18 Z. Zhang, S. Chen and S. Jiang, Biomacromolecules, 2006, 7, 3311-3315.

19 B. V. Slaughter, S. S. Khurshid, O. Z. Fisher, A. Khademhosseini and N. A. Peppas, Adv. Mater., 2009, 21, 3307-3329.

20 T. Goda, R. Matsuno, T. Konno, M. Takai and K. Ishihara, J Biomed Mater Res Part 
B: Appl Biomater., 2009, 89B, 184-190.

21 L. R. Carr, Y. Zhou, J. E. Krause, H. Xue and S. Jiang, Biomaterials, 2011, 32, 6893-6899.

22 Z. Zhang, T. Chao, L. Liu, G. Cheng, B. D. Ratner and S. Jiang, J. Biomater. Sci., 2009, 20, 1845-1859.

23 N. Ishiyama, T. Moro, T. Ohe, T. Miura, K. Ishihara, T. Konno, T. Ohyama, M.

Kimura, M. Kyomoto, T. Saito, K. Nakamura, H. Kawaguchi, J. Bone. Joint. Surg. Am., $2011,93,142-149$

24 S. Naficy, H. R. Brown, J. M. Razal, G. M. Spinks and P. G. Whitten, Aust. J. Chem., 2011, 64, 1007-1025.

25 J. P. Gong, Soft Matter, 2010, 6, 2583-2590.

26 L. Shi, R. Aid, C. Le Visage and S. Y. Chew, Macromol. Biosci., 2012, 12, 395-401. 27 W. Liu, C. Deng, C. R. McLaughlin, P. Fagerholm, N. S. Lagali, B. Heyne, J. C. Scaiano, M. A. Watsky, Y. Kato, R. Munger, N. Shinozaki, F. Li and M. Griffith, Biomaterials, 2009, 30, 1551-1559.

28 T. Sakai, T. Matsunaga, Y. Yamamoto, C. Ito, R. Yoshida, S. Suzuki, N. Sasaki, M. Shibayama and U. Chung, Macromolecules, 2008, 41, 5379-5384.

29 K. Haraguchi, Polym. J., 2011, 43, 223-241. 
30 J. P. Gong, Y. Katsuyama, T. Kurokawa and Y. Osada, Adv.Mater., 2003, 15, $1155-1158$.

31 M. Huang, H. Furukawa, Y. Tanaka, T. Nakajima, Y. Osada and J. P. Gong, Macromolecules, 2007, 40, 6658-6664.

32 T. Nakajima, H. Sato, Y. Zhao, S. Kawahara, T. Kurokawa, K. Sugahara and J. P. Gong, Adv. Funct. Mater., 2012, 22, 4426-4432.

33 S. P. Obukhov, M. Rubinstein and R. H. Colby, Macromolecules, 1994, 27, 3191-3198.

34 M. Rubinstein, R. H. Colby, A. V. Dobrynin and J.-F. Joanny, Macromolecules 1996, 29, 398-406.

35 P. J. Flory, Principles of Polymer Chemistry, Cornell University Press, 1953.

36 D. N. Schulz, D. G. Peiffer, P. K. Agarwal, J. Larabee, J. J. Kaladas, L. Soni, B. Handwerker and R. T. Garner, Polymer, 1986, 27, 1734-1742.

37 L. Chen, Y. Honma, T. Mizutani, D. J. Liaw, J. P. Gong and Y. Osada, Polymer, 2000, 41, 141-147.

38 A. Ohsugi, H. Furukawa, A. Kakugo, Y. Osada and J. P. Gong, Macromol. Rapid. Commun., 2006, 27, 1242-1246.

39 Y. Tanaka, R. Kuwabara, Y.-H. Na,. T. Kurokawa, J. P. Gong and Y. Osada, J. Phys. 
Chem. B 2005, 109, 11559-11562.

40 S. Liang, Q. M. Yu, H. Yin, Z. L. Wu, T. Kurokawa and J. P. Gong, Chem.

Commun., 2009, 48,7518-7520.

41 Y. Tanabe, K. Yasuda, C. Azuma, H. Taniguro, S. Onodera, A. Suzuki,Y. M. Chen, J. P. Gong and Y. Osada, J Mater Sci: Mater Med., 2008, 19, 1379-1387.

42 K. Yasuda, N. Kitamura, J. P. Gong, K. Arakaki, H. J. Kwon, S. Onodera, Y. M.

Chen, T. Kurokawa, F. Kanaya, Y. Ohmiya and Y. Osada, Macromol. Biosci.,2009, 9, 307-316.

43 Y. Tamada and Y. Ikada, J. Colloid Interface Sci., 1993, 155, 334-339

44 H. Kitano, S. Tada, T. Mori, K. Takaha, M. Gemmei-Ide, M. Tanaka, M. Fukuda and Y. Yokoyama, Langmuir, 2005, 21, 11932-11940

45 H. Kitano, K. Nagaoka, S. Tada, M. Gemmei-Ide and M. Tanaka, Macromol. Biosci., $2008,8,77-85$

46 J. J. Yang, Y. M. Chen, J. F. Liu, T. Kurokawa and J. P. Gong, Tissue Eng., part A., 2010, 16, 2529-2540.

47 Y. M. Chen, M. Tanaka, J. P. Gong, K. Yasuda, S. Yamamoto, M. Shimomura and Y. Osada, Biomaterials, 2007, 28, 1752-1760. 
(a)<smiles>C=C(C)C(=O)OCC[N+](C)(C)CC(=O)[O-]</smiles>

(b)

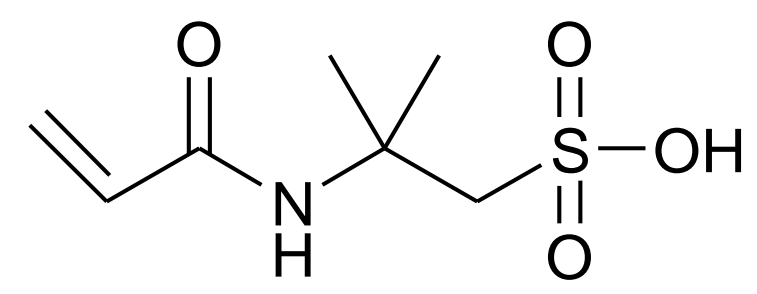

Scheme 1. Chemical structures of monomers used in this work. (a) $\mathrm{N}$-(carboxymethyl)-N, N-dimethyl-2-methacryloyloxy) ethanaminium, inner salt (CDME). (b) 2-acrylamido-2-methyl propanesulfonic acid. 


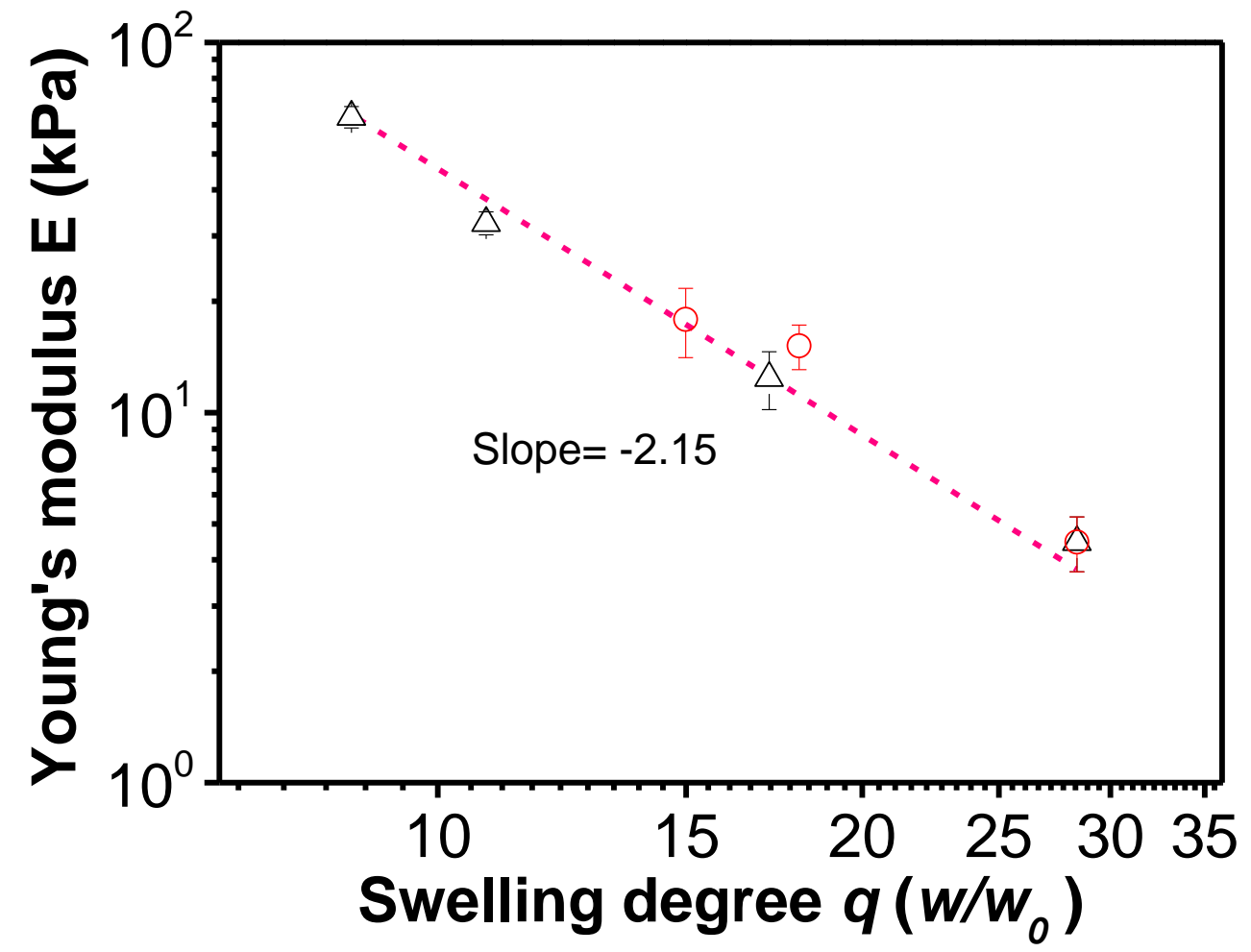

Figure 1. Relationship of the degree of swelling $q$ and Young's modulus $E$ of the PCDME single network hydrogel with different formulation. Red circles: different CDME concentration (from left to right: SN3-0.3, SN2-0.3, SN1-0.3). Black triangles: different cross-linker density (from left to right: SN1-6, SN1-3, SN1-1, SN1-0.3). 
(a)

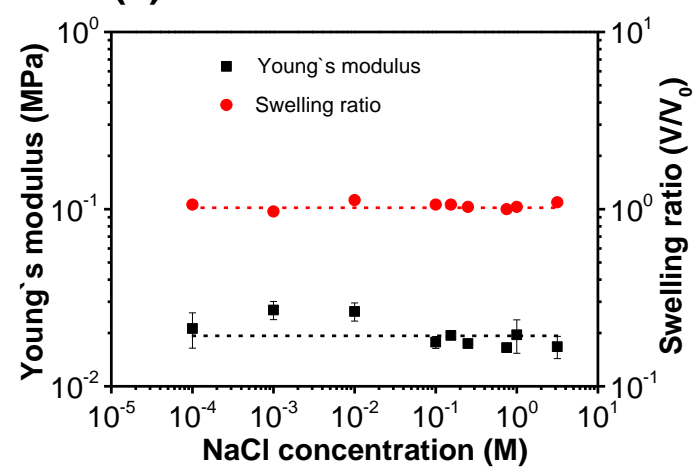

(b)

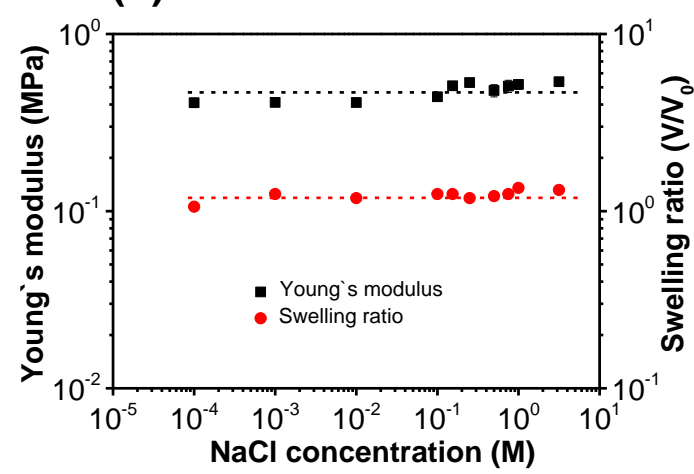

Figure 2. Effect of $\mathrm{NaCl}$ concentration on the Young's modulus $E$ and swelling ratio (V/ $\mathrm{V}_{0}$ ) of PCDME hydrogel (SN1-1) (a) and PAMPS/PCDME DN hydrogel (DN2) (b).

$\mathrm{V}$ and $\mathrm{V}_{0}$ were volumes of sample in $\mathrm{NaCl}$ aqueous solution and in water, respectively. 


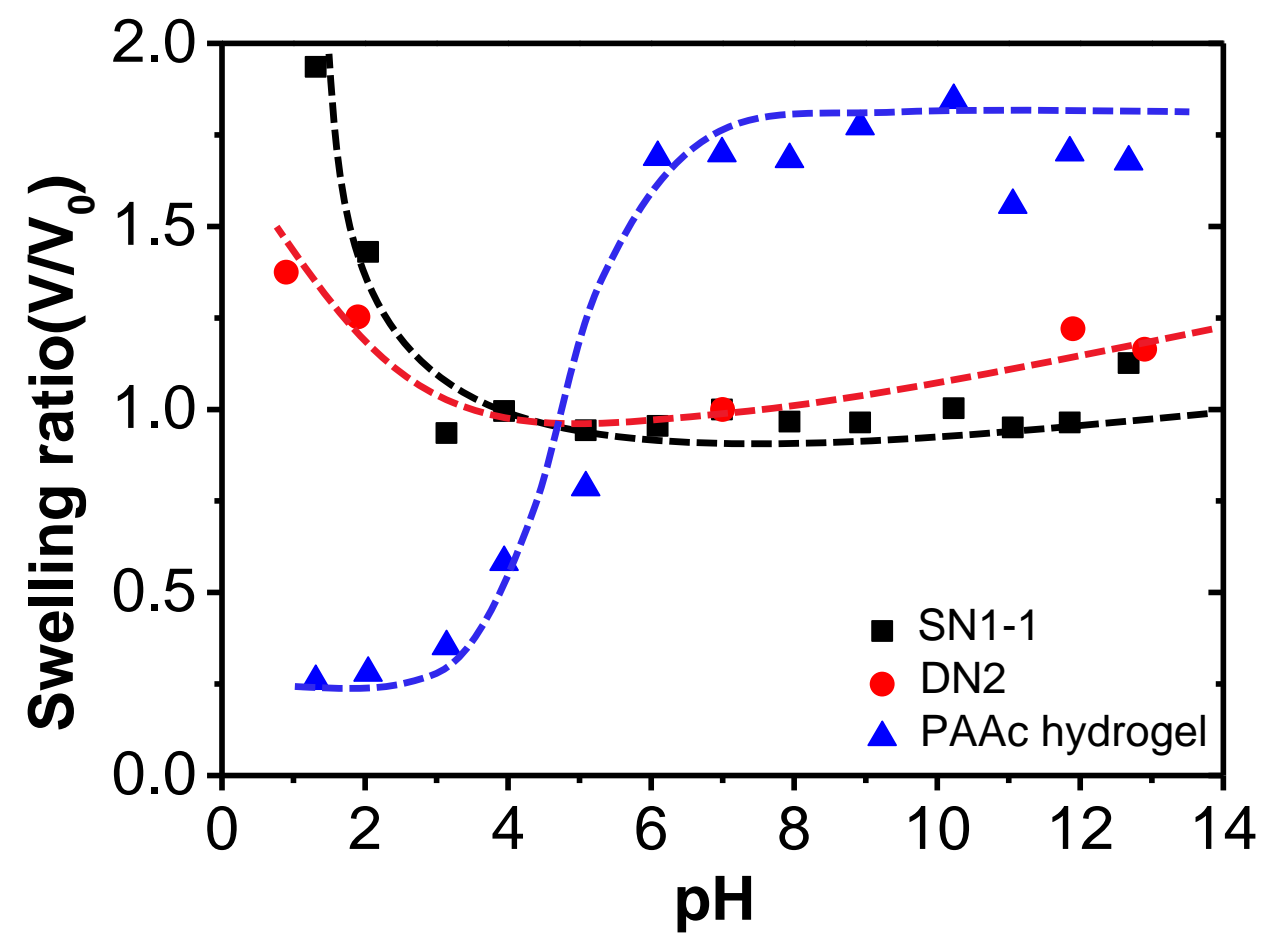

Figure 3. Effect of $\mathrm{pH}$ on the swelling ratio $\left(\mathrm{V} / \mathrm{V}_{0}\right)$ of PCDME hydrogel (SN1-1) and PAMPS/PCDME DN hydrogel (DN2). As a comparison, the result of a PAAc hydrogel(AAc-MBAA-Initiator:1M-4mol\%-0.1mo\%) is also shown. $\mathrm{V}$ and $\mathrm{V}_{0}$ are volumes of samples in various $\mathrm{pH}$ and in water, respectively. 


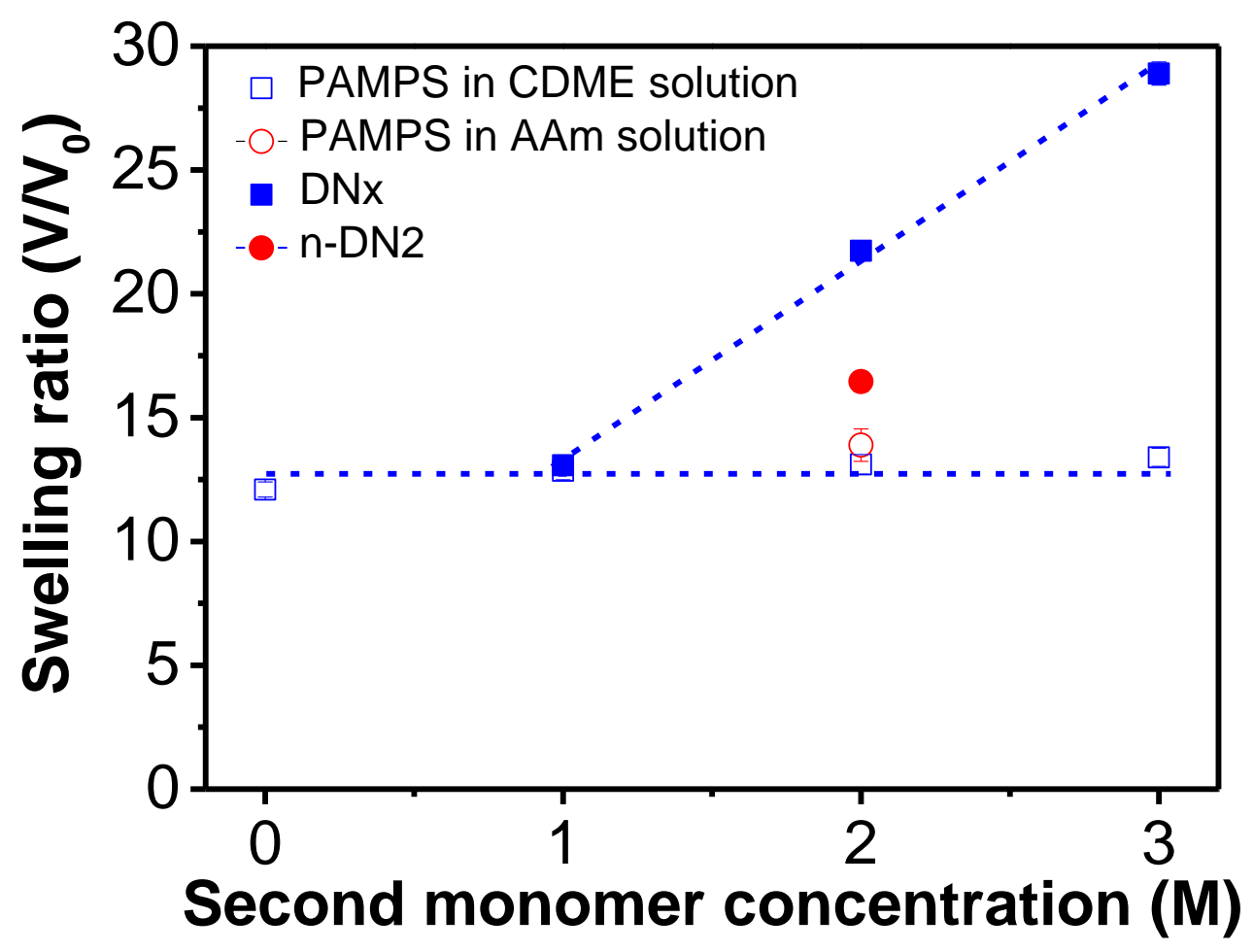

Figure 4. Swelling ratios $\left(\mathrm{V} / \mathrm{V}_{0}\right)$ of PAMPS hydrogel in precursor solution of the second network with various CDME concentrations (open squares), and swelling ratios of the corresponding PAMPS/PCDME DN hydrogels (DNx) in pure water (solid squares). $\mathrm{V}_{0}$ is the volume of PAMPS at the as-prepared state, and V is that of PAMSP gels in the $2^{\text {nd }}$ network precursor solution or DN gel (solid points) in water. For comparison, the results of AAm as the second network component are also shown. 


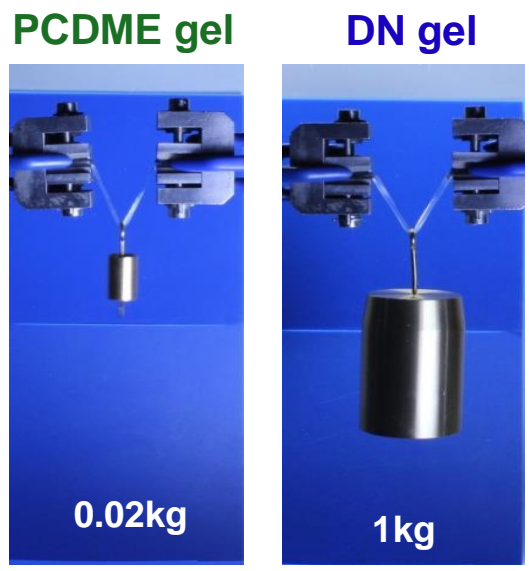

Figure 5. Images of a single PCDME hydrogel (SN1) and a tough PAMPS/PCDME DN hydrogel (DN3) sustaining load of $0.02 \mathrm{kgf}$ and $1 \mathrm{kgf}$, respectively. (Sample size: $\left.20 \times 4 \times 2.5 \mathrm{~mm}^{3}\right)$ 
(a)

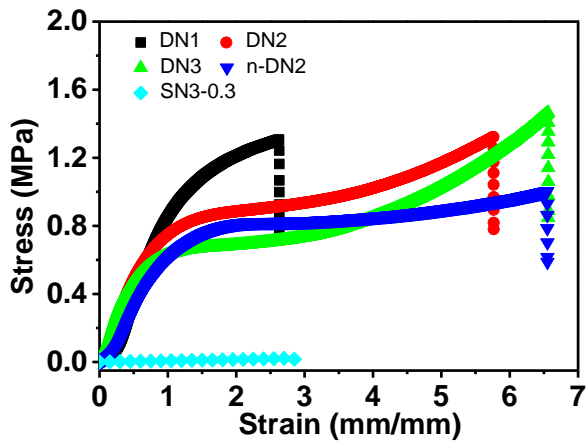

(b)

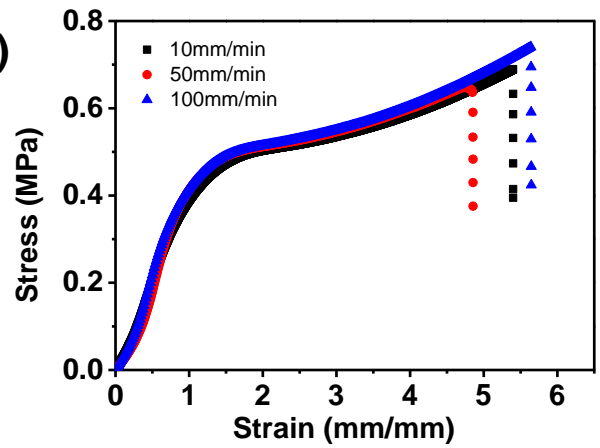

(a')

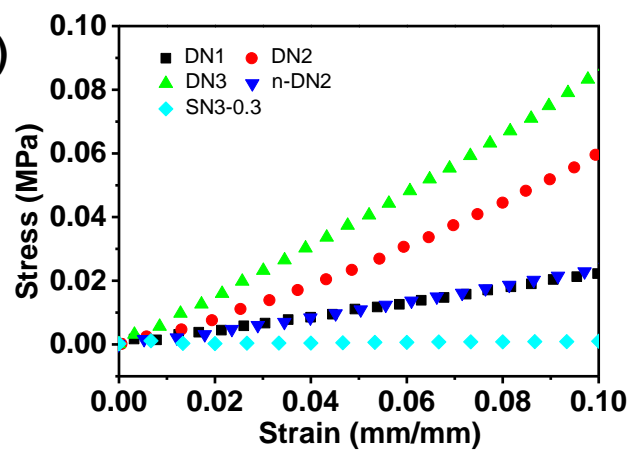

(c)

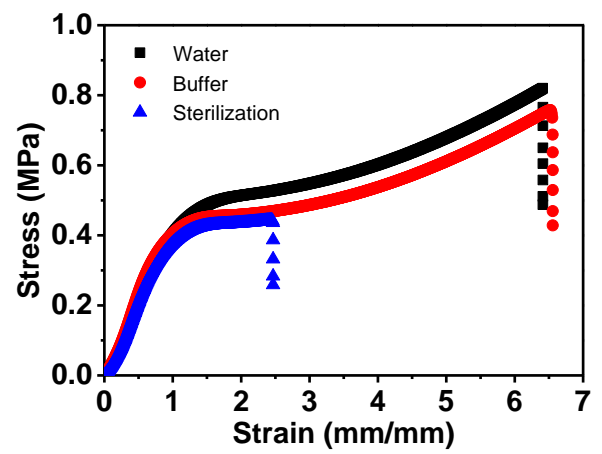

Figure 6. (a)Tensile stress-strain behaviors of PAMPS/PCDME DN(DNx) hydrogels prepared at various CDME concentrations. Results of normal PAMPS/PAAm DN hydrogel(n-DN2) and the PCDME single network hydrogel (SN3-0.3) are also shown for comparison. (a') zoom at low strains of the tensile stress-strain curves of (a). (b) Tensile stress-strain behaviors of PAMPS/PCDME DN hydrogel (DN3) measured at different velocities. (c) Tensile stress-strain behaviors of PAMPS/PCDME DN hydrogel (DN3) before and after HEPES buffer and sterilization treatments. 
(a)

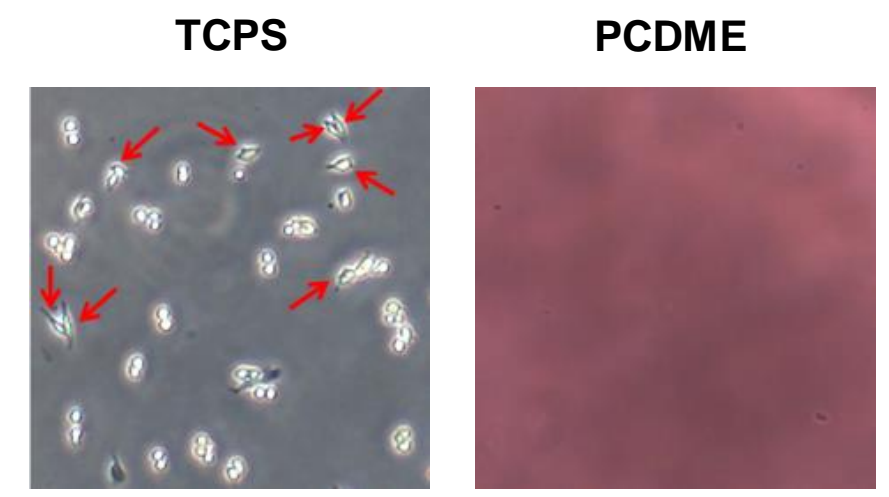

PAMPS/PCDME DN

(b)

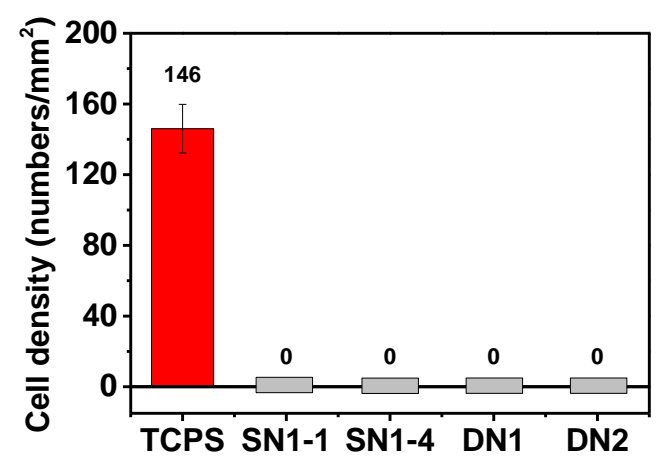

Figure 7. (a) Morphology of macrophages on the tissue culture polystyrene (TCPS),

PCDME hydrogel (SN1-1), and PAMPS/PCDME DN hydrogel(DN1) after seeding for 24h. Red arrows indicate macrophage adhered on the TCPS surface. (b)Number density of macrophage adhered on TCPS and various PDCME hydrogels and PAMPS/PCDME DN hydrogels after seeding for $24 \mathrm{~h}$. 
Table 1. Sample codes and properties of PCDME single network hydrogels.

\begin{tabular}{|c|c|c|c|c|c|c|c|}
\hline $\begin{array}{l}\text { Sample } \\
\text { code }\end{array}$ & $\begin{array}{l}\mathrm{C}_{\mathrm{CDME}} \\
(\mathrm{M})\end{array}$ & $\begin{array}{l}\mathrm{C}_{\mathrm{MBAA}} \\
(\mathrm{mol} \%)\end{array}$ & $\begin{array}{l}\text { Degree of } \\
\text { swelling } \\
q\end{array}$ & $\begin{array}{l}\text { "Young's } \\
\text { modulus } \\
E(\mathrm{kPa})\end{array}$ & ${ }^{* * *} \mathrm{~N}$ & $\begin{array}{l}\text { "Fracture } \\
\text { stress } \\
(\mathrm{kPa})\end{array}$ & $\begin{array}{l}\text { *Fracture } \\
\text { strain } \\
(\mathrm{mm} / \mathrm{mm})\end{array}$ \\
\hline SN1-0.3 & 1.0 & 0.3 & $28.4 \pm 0.1$ & $4.5 \pm 0.8$ & 253 & $4.3 \pm 0.0$ & $0.5 \pm 0.0$ \\
\hline SN1-1 & 1.0 & 1 & $17.2 \pm 0.2$ & $12 \pm 2.2$ & 158 & $14 \pm 0.0$ & $0.4 \pm 0.0$ \\
\hline SN1-3 & 1.0 & 3 & $10.8 \pm 0.2$ & $33 \pm 2.3$ & 93 & $43 \pm 0.0$ & $0.4 \pm 0.2$ \\
\hline SN1-6 & 1.0 & 6 & $8.7 \pm 0.1$ & $63 \pm 4.2$ & 61 & 295 & 0.8 \\
\hline SN2-0.3 & 2.0 & 0.3 & $18.0 \pm 0.1$ & $15 \pm 2.1$ & 120 & 247 & 1.0 \\
\hline SN3-0.3 & 3.0 & 0.3 & $15.0 \pm 0.1$ & $18 \pm 3.8$ & 121 & 605 & 1.0 \\
\hline
\end{tabular}

*: Compression results.

${ }^{* *} N$ : The number of CDME units between the crosslinking points. 
Table 2. Sample codes and properties of PAMPS/PCDME DN hydrogels.

\begin{tabular}{|c|c|c|c|c|c|c|c|}
\hline $\begin{array}{l}\text { Sample } \\
\text { code }\end{array}$ & $\begin{array}{l}\mathrm{C}_{\mathrm{CDME}} \\
(\mathrm{M})\end{array}$ & $\begin{array}{l}\text { Water } \\
\text { Content } \\
c(w t \%)\end{array}$ & $\begin{array}{l}{ }^{*} \text { Composition } \\
\text { ratio }\left(2^{\text {nd }} / 1^{\text {st }}\right) \\
(\mathrm{mol} / \mathrm{mol})\end{array}$ & $\begin{array}{l}\text { Young's } \\
\text { Modulus } \\
\text { E (MPa) }\end{array}$ & $\begin{array}{l}{ }^{* *} \text { Fracture } \\
\text { Stress } \\
(\mathrm{MPa})\end{array}$ & $\begin{array}{l}{ }^{* *} \text { Fracture } \\
\text { strain } \\
(\mathrm{mm} / \mathrm{mm})\end{array}$ & $\begin{array}{l}{ }^{* *} \text { Work of } \\
\text { extension } \\
\left(\mathrm{MJ} / \mathrm{m}^{3}\right)\end{array}$ \\
\hline DN1 & 1.0 & 82.0 & 10 & $0.2 \pm 0.1$ & $1.2 \pm 0.1$ & $2.2 \pm 0.4$ & $0.9 \pm 0.3$ \\
\hline DN2 & 2.0 & 79.4 & 25 & $0.8 \pm 0.2$ & $1.2 \pm 0.2$ & $5.1 \pm 1.1$ & $2.1 \pm 0.7$ \\
\hline DN3 & 3.0 & 78.7 & 38 & $0.9 \pm 0.2$ & $1.4 \pm 0.1$ & $6.0 \pm 0.8$ & $2.4 \pm 0.6$ \\
\hline n-DN2 & 2.0 & 89.5 & 20 & $0.2 \pm 0.0$ & $0.9 \pm 0.1$ & $4.9 \pm 1.6$ & $2.9 \pm 0.5$ \\
\hline & (AAm) & & & & & & \\
\hline
\end{tabular}

${ }^{*}$ :Composition ratio of the $2^{\text {nd }}$ network to the $1^{\text {st }}$ network was determined by elemental analysis.

**: Tensile results. 
Table 3. Degree of swelling and contact angle of hydrogels and TCPS.

\begin{tabular}{lll}
\hline Sample code & Degree of swelling $q$ & Contact angle $\theta\left(^{\circ}\right)$ \\
\hline SN1-1 & 17.2 & 11.4 \\
SN1-3 & 10.8 & 19.9 \\
DN1 & 4.94 & 29.5 \\
DN2 & 5.40 & 31.0 \\
DN3 & 4.95 & 37.5 \\
PAMPS & 175 & 14.3 \\
TCPS & - & 69.0 \\
\hline
\end{tabular}

